\title{
Clinical features of sodium-taurocholate cotransporting polypeptide deficiency in pediatric patients: case series and literature review
}

\author{
Ting-Ting Zou, Yu Zhu, Chao-Min Wan, Qiong Liao \\ Department of Pediatric Infectious Diseases, West China Second University Hospital, Key Laboratory of Birth Defects and Related Diseases of \\ Women and Children (Sichuan University), Ministry of Education, Chengdu, China \\ Correspondence to: Qiong Liao, MD. Department of Pediatric Infectious Diseases, West China Second University Hospital, Key Laboratory of Birth \\ Defects and Related Diseases of Women and Children (Sichuan University), Ministry of Education, Chengdu 610041, China. Email: 33765410@qq.com.
}

\begin{abstract}
Sodium-taurocholate cotransporting polypeptide (NTCP) deficiency is a newly reported hereditary bile acid metabolic disease. Here we describe the clinical characteristics of 12 cases of pediatric NTCP deficiency, as well as review 60 previously reported cases in the literature in order to provide better guidance for pediatricians. The clinical records, laboratory and imaging data were collected of 12 cases who were treated at the pediatric infectious disease department of the West China Second University Hospital of Sichuan University, China, from December 2018 to July 2020. PubMed and Wanfang databases were searched and 11 studies including 60 pediatric NTCP deficiency patients from January 2015 to November 2020 were retrieved. In our center, there were 4 girls and 8 boys, with a median age at admission of 9.9 months (range, 2.2 to 70 months). Six patients $(50 \%)$ had prolonged neonatal jaundice. All of the patients $(12 / 12 ; 100 \%)$ had normal growth and development. The reason for the first visit was prolonged neonatal jaundice (4/12, 33.3\%), non-liver related diseases $(6 / 12,50 \%)$ and routine checkup (2/12, 16.7\%). Hypercholanemia was documented in 12/12 (100\%), elevated aspartate aminotransferase (AST) in 6/12 (50\%), and elevated alanine aminotransferase (ALT) in 1/12 (8.3\%). All of the patients (12/12; 100\%) had homozygous mutations of c.800C>T in SLC10A1. Sixty patients (22 girls and 38 boys) were included in the literature review; 36 (60\%) had hyperbilirubinemia after 1 month. The reasons for testing for hypercholanemia were identified in 47/60 cases, and included prolonged neonatal jaundice and neonatal transient cholestasis in 26 (26/47, 55.3\%); non-liver related diseases in 14 (14/47, 29.8\%); routine medical examination in $3(3 / 14,6.4 \%)$; volunteer recruitment in $1(1 / 14,7.1 \%)$; dark urine in $1(1 / 47$, $2.1 \%)$. Hypercholanemia was confirmed in 60/60 (100\%); 31 (51.7\%) had elevated AST, and 10 (16.7\%) had elevated ALT. Among 59 Chinese patients, 52 (88.1\%) had homozygous mutations of c.800C>T in SLC10A1. The most common symptom of pediatric NTCP deficiency is jaundice. NTCP deficiency can also be detected during routine check-ups. The common biochemical features are hypercholanemia and elevated AST. Screening for c.800C>T mutation in SLC10A1 is useful for primary genetic screening in Chinese infants with persistent hypercholanemia after infectious, structural, and immunological factors are excluded.
\end{abstract}

Keywords: Sodium-taurocholate cotransporting polypeptide (NTCP); hypercholanemia; pediatric; case series

Submitted Nov 03, 2020. Accepted for publication Feb 22, 2021.

doi: $10.21037 / \mathrm{tp}-20-360$

View this article at: http://dx.doi.org/10.21037/tp-20-360 


\section{Introduction}

Sodium-taurocholate cotransporting polypeptide (NTCP) is encoded by the solute carrier family 10 member 1 (SLC10A1) gene with a total length of $23 \mathrm{kbp}$, localized in chromosome $14 \mathrm{q} 24.2$, which contains 5 exons. The protein product NTCP is composed of 349 amino acid residues, with a molecular weight of $38 \mathrm{kDa}$ (1). NTCP is localized at the basolateral membrane of hepatocytes. It is a sodiumdependent transporter that is involved in the transport of bile acids from the blood to the hepatocytes in order to maintain the uninterrupted enterohepatic circulation of bile salts, and its deficiency leads to elevated levels of circulating bile acids (hypercholanemia). The clinical and biochemical influences of hypercholanemia are not fully understood. As of November 2020, a total of 60 cases of pediatric NTCP deficiency had been reported in 11 studies. The common feature is hypercholanemia without obvious symptoms and the mode of inheritance is autosomal recessive. However, the clinical features need to be further defined, especially in pediatric patients, in order to promote early recognition and avoid unnecessary intervention and anxiety. Herein, we reported 12 cases of pediatric NTCP deficiency to determine the clinical features and review the previous cases in the literature to identify additional common characteristics. We present the following case in accordance with the CARE reporting checklist (available at http:// dx.doi.org/10.21037/tp-20-360).

\section{Case presentation}

\section{Methods}

\section{Patients}

A retrospective, observational case series study involving 12 children with NTCP deficiency and SLC10A1 gene mutation was conducted at the pediatric infectious disease department of the West China Second University Hospital of Sichuan University, China. The clinical research registry number is ChiCTR2000040305. All procedures performed in studies involving human participants were in accordance with the ethical standards of the institutional and/or national research committee(s) and with the Helsinki Declaration (as revised in 2013). Written informed consent was obtained from the patient's legal guardian.

All the patients under the age of 18 years who were hospitalized for persistent hypercholanemia and diagnosed by gene sequencing with homozygous mutations of SLC10A1 from December 2018 to July 2020 were included.
Patients with infectious, structural, and immunological cholestasis were excluded. The clinical records, laboratory data and imaging data were collected retrospectively and analyzed. The follow-up duration ranged from 1 to 45.2 months.

\section{Data collection and literature retrieval}

Baseline patient characteristics including sex, date of admission, age and weight at admission, gestational age, birth weight, reason for visit, family history, and past history and clinical presentation, including the presence of jaundice, pruritus, and steatorrhea, color of stools, and developmental milestones were examined. Additional laboratory data included alanine aminotransferase (ALT), aspartate aminotransferase (AST), $\gamma$-glutamyl transpeptidase (GGT), alkaline phosphatase (ALP), total bilirubin (TB), direct bilirubin (DB), albumin (ALB), total bile acid (TBA), 25-hydroxy vitamin $\mathrm{D}(\mathrm{VD})$, and blood ammonia $\left(\mathrm{NH}_{3}\right)$ levels, results of viral panels [hepatitis A, B, C, and E, human immunodeficiency virus (HIV), Epstein-Barr virus (EBV), and cytomegalovirus], and autoimmune hepatitis screen. Imaging data included fasted transabdominal ultrasound and genetic data included next generation sequencing (NGS) for inherited metabolic liver disorders, including 573 related genes (Table S1; website: https:// pan.baidu.com/s/1-45A2hrGNnwYmGCIynkHdg; code: rmjf) and verification of mutations by Sanger sequencing. The PubMed and Wanfang databases were searched using (sodium taurocholate cotransporting polypeptide or sodium-taurocholate cotransporting polypeptide or $\mathrm{Na}$-taurocholate cotransporting polypeptide or NTCP) and (child or children or pediatric or pediatrics). Clinical features and laboratory data of previous NTCP deficiency patients were collected from the relevant pediatric NTCP deficiency studies.

\section{Statistical analysis}

Statistical analysis was performed with SPSS software, version 23.0 (IBM Corp., New York, NY, USA).

\section{Results}

\section{Patient characteristics}

A total of 12 patients, 4 girls and 8 boys, sorted by admission date as Patient 1 (P1) to Patient 12 (P12), were included. All of them are Chinese. Serum TBA is routinely measured in liver function tests in some hospitals in China, and liver function tests are routinely performed 
for hospitalized patients and for pre-school children before entering school. As our hospital is a tertiary pediatric center, hypercholanemia was firstly detected in the patients at their local hospitals. The median age of the cohort at the time of admission to our center was 9.9 months (range, 2.2 to 70 months). Patients P1-5, $\mathrm{P} 7-\mathrm{P} 9$, and P11-P12 were full term. The gestational ages of patients P 6 and P10 were 34 weeks plus 2 days and 36 weeks plus 3 days. The birth weights ranged from 2.10 to $4.20 \mathrm{~kg}$ (median: 3.15, IQR: 0.85 ). The birth weights were from the third to the ninety seventh percentile around the median for the same gestational age and sex according to the 2015 percentile curves for Chinese neonatal birth weight for gestational age. The mothers of P1 and P10 had intrahepatic cholestasis during pregnancy. No other relevant pregnancy history was found for the other mothers. P4 was adopted by the present adoptive parents, and the history of pregnancy and delivery could not be obtained. P1, P3, P4, P5, P6, and $\mathrm{P} 10$ had prolonged neonatal jaundice, defined as lasting for more than 2 weeks in full term neonates and more than 1 month in pre-term neonates. P4 underwent pyloromyotomy and cholecystostomy for recurrent vomiting and prolonged cholestasis. The median weight at admission was $8.40 \mathrm{~kg}$ (range, 6.80 to $20.00 \mathrm{~kg}$, IQR 5.00). The weights at admission ranged from the third to the ninetieth percentiles around the median for the same age and sex according to the 2009 percentile curves for weight in Chinese children aged 0 to 3 and 2 to 18 years. The evaluation of motor and physical development of all the patients was normal. No symptom of pruritus or steatorrhea was recorded. No obvious abnormalities were mentioned in the physical examinations.

\section{Clinical features}

The reasons for the first visit to the local hospital was jaundice (4/12, 33.3\%), pneumonia (4/12, 33.3\%), preterm birth $(1 / 12,8.3 \%)$, acute suppurative tonsillitis $(1 / 12$, $8.3 \%)$, and routine medical examination for enrollment to kindergarten $(2 / 12,16.7 \%)$. Laboratory abnormalities at the local hospitals included hypercholanemia, hyperbilirubinemia and abnormal transaminase levels, especially AST. At the time of admission to our center, all of the patients $(12 / 12,100 \%)$ presented with persistent hypercholanemia, 6/12 presented elevated AST levels, with only 1 showing a $>2$-fold increase from the upper limit (normal range: 0 to $40 \mathrm{U} / \mathrm{L}$ ), and 1/12 had elevated ALT, to $<2 \times$ the upper limit value (normal range, 0 to $49 \mathrm{U} / \mathrm{L}$ ).
Two patients had hyperbilirubinemia when admitted to our center. Four patients (P1, P4, P11, P12) were tested for serum 25-hydroxy vitamin D level; 1 of them showed $<20 \mathrm{ng} / \mathrm{mL}$ (normal range, 20 to $100 \mathrm{ng} / \mathrm{mL}$ ). Blood $\mathrm{NH}_{3}$ levels were measured in 5 patients and were slightly above normal (5.88 to $35.3 \mu \mathrm{mol} / \mathrm{L})$ in 3 . The GGT levels were normal except in 2 patients (P4, P5) at early infancy (Table 1). The other items of the liver function test, including ALP, ALB, globulin, triglycerides, and total cholesterol, were at normal levels.

The follow-up duration ranged from 1 to 45.2 months (median 13 months). The TBA levels fluctuated and were elevated without regularity except in P6, who had a normal TBA level at the last follow-up (Table S2; website: https:// pan.baidu.com/s/1nJC1czxVqubn8RX9JxeDBw; code: dgn3). Growth and development were always normal for all of the patients and no other comorbidities were found.

\section{Genetic data}

Homozygous mutations of SLC10A1(c.800C >T) were detected in all the patients by next-generation sequencing and confirmed by Sanger sequencing. Homozygous mutations of SLC10A1(c.800C > T) were also documented in the mother of $\mathrm{P} 1$ and the parents of $\mathrm{P} 10$, and the other parents had heterozygous mutations. The father of P10 was asymptomatic, while the mothers of $\mathrm{P} 1$ and $\mathrm{P} 10$ both had intrahepatic cholestasis during pregnancy. P1 and P6 also showed homozygous mutations of UGT1A1, while P5 had compound heterozygous mutations of UGT1A1, P10 had heterozygous mutation of UGT1A1, and P3 had heterozygous mutation of SLC25A13 (Table 1). The other genes correlated with bile acid and bilirubin metabolism were negative.

\section{Literature review}

Eleven studies including 60 pediatric patients with NTCP deficiency from January 2015 to November 2020 were retrieved from PubMed and Wanfang databases (2-12). The first patient was Afghan, who was referred to the hospital for developmental delay. The other 59 patients were Chinese, and no growth or developmental delays were reported except two patients reported by Yan et al. (12). The female to male ratio was 22:38. Thirty-six patients $(60 \%)$ had hyperbilirubinemia after 1 month of age and 3 were found to have citrin deficiency. Reasons for checking TBA were documented in 47/60 patients and included prolonged neonatal jaundice and neonatal transient cholestasis (26/47, $55.3 \%)$; non-liver related diseases $(14 / 47,29.8 \%)$; routine 


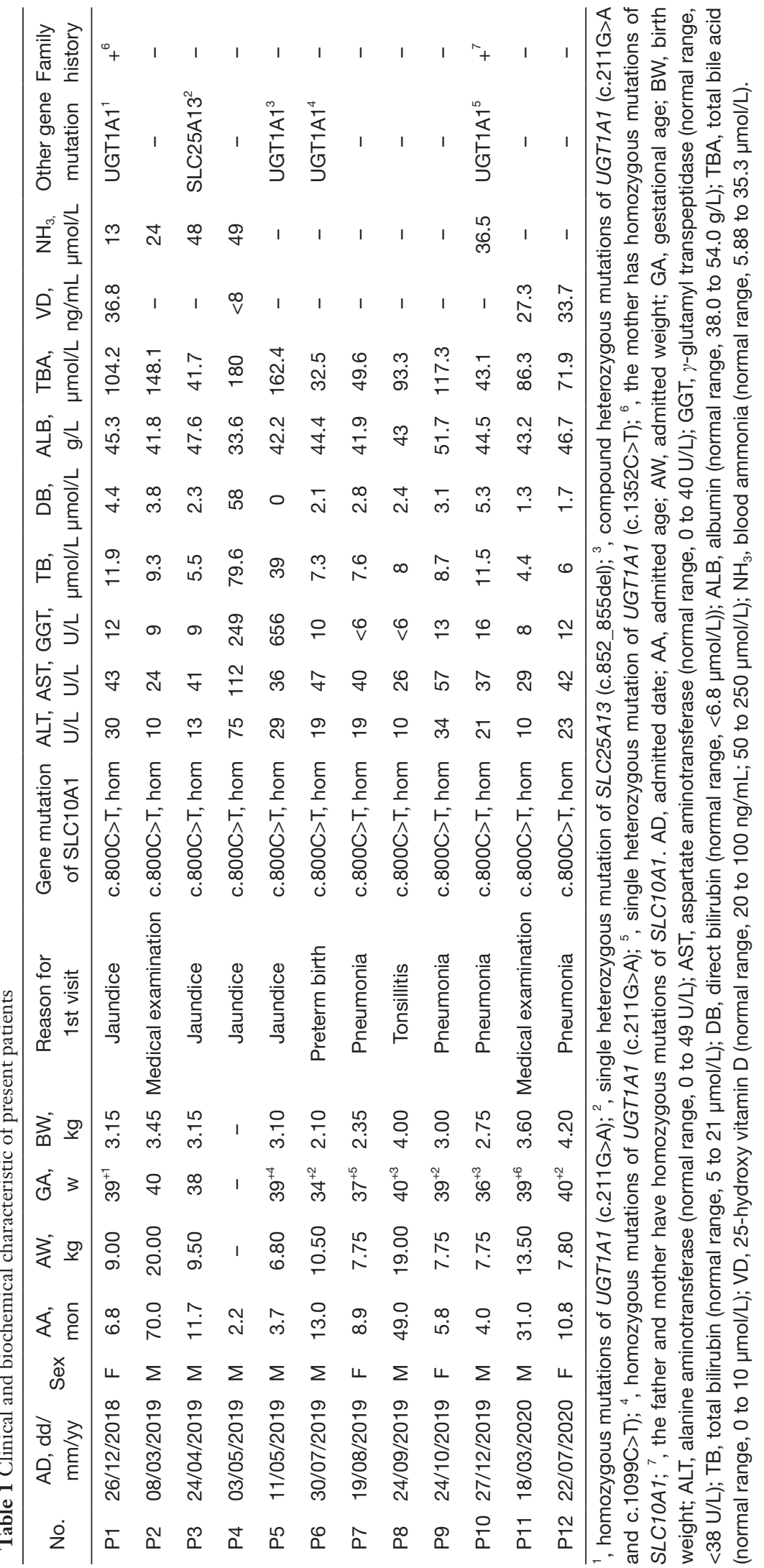


medical examination $(3 / 14,6.4 \%)$; volunteer recruitment $(1 / 14,7.1 \%)$; and dark urine $(1 / 47,2.1 \%)$. Serum biochemistry revealed hypercholanemia (60/60, 100\%), elevated AST (31/60, 51.7\%) (normal range, 0 to $40 \mathrm{U} / \mathrm{L}$ ), and elevated ALT (10/60, 16.7\%) (normal range, 0 to $49 \mathrm{U} / \mathrm{L})$. However, only 10 patients had $>2$-fold elevations of AST, and 6 patients had >2-fold elevations of ALT. The 25-hydroxy vitamin D levels were checked in 19 patients and were $<20 \mathrm{ng} / \mathrm{mL}$ (normal range, 20 to $100 \mathrm{ng} / \mathrm{mL}$ ) in 6. The long-term follow-up revealed that the first Afghan patient presented developmental delay but normal growth of height and weight. The patient also presented mild liver dysfunction, including mildly elevated ALT, AST, and TB. The fat-soluble vitamins were at a normal or slightly lower level. The TBA level declined with increasing age (13).

We reviewed and summarized the baseline information and clinical characteristics of the patients in order to identify common features of NTCP deficiency. The results are shown in Table 2.

\section{Discussion}

NTCP deficiency is a novel condition that was first described in 2015 (2). Here we report 12 cases of pediatric NTCP deficiency from our center in order to provide additional information about this condition. In this series, the youngest age at diagnosis was 2.2 months, and the oldest was 70 months. More boys than girls were affected, and the reasons for the first visit were heterogeneous, with the most common being jaundice. In the younger infants, hypercholanemia was detected during diagnostic evaluation for prolonged neonatal jaundice or other acute infectious diseases and in the preschool children, hypercholanemia was detected by routine medical examination. Besides hypercholanemia, which was present in $12 / 12$ patients (100\%), the most common biochemical feature was elevated AST (50\%), which was usually slight ( $<2 \times$ normal in $83.3 \%)$. Only 2 patients, both between the ages of 2 and 4 months, had hyperbilirubinemia, and no hyperbilirubinemia was found among patients older than 4 months. Homozygous mutations of SLC10A1(c.800C>T) were documented in all patients, and in 5/12 these mutations were combined with other gene mutations (including UGT1A1 and SLC25A13) that could influence the metabolism of bilirubin $(14,15)$. All of the patients in this series had normal growth and development.

The general characteristics and the reason for the first visit in our series were in agreement with those of the previous studies. Most of the previously reported patients had normal growth and development besides the first Afghan patient and two Chinese patients reported by Yan et al. $(2,12)$. However, the follow-up of the Afghan patient suggested that the developmental delay might not be directly related to the chronic hypercholanemia (16).The cause of the developmental delay remains to be explored. The most common biochemical features of the previous cases included hyperbilirubinemia as well as elevated AST, which differed from our series, probably because $11 / 12$ patients in our group had hypercholanemia for at least 3 months (and up to 2 years) before they were referred to our hospital, and by the time they were admitted to our center, bilirubin levels had decreased to normal. This suggested that the hyperbilirubinemia was selflimiting. Four of 6 patients $(66.7 \%)$ at our center who had prolonged neonatal jaundice displayed other gene mutations that could influence bilirubin metabolism, especially in early infancy. Thus, whether or not NTCP deficiency can influence the metabolism of bilirubin needs further study for clarification on the molecular level. The elevation of AST is not matched to that of ALT, which also needs to be investigated further. About 30.4\% (7/23) of the present and previous patients who underwent 25-hydroxy vitamin D testing had low (<20 ng/mL) 25 -hydroxy vitamin D levels. However, postnatal vitamin $\mathrm{D}$ supplementation is routinely recommended in Chinese infants, and 25-hydroxy vitamin D levels may vary based on different amounts of supplementation. Because the other fat soluble vitamins were not measured, the effect of NTCP deficiency on the absorption of fat-soluble vitamins remains to be discovered.

When NTCP deficiency mimics typical infantile cholestasis, it may cause erroneous suspicion of biliary atresia or other bile duct abnormality, prompting invasive examinations, such as endoscopic retrograde cholangiopancreatography and bile duct exploration, before the correct diagnosis is made. In addition, before the diagnosis is confirmed, some patients will take drugs to protect the hepatocytes and promote bile excretion that are not needed for NTCP deficiency. The parents may be unduly distressed and the patients will frequently be taken to multiple medical institutions. Thus, the early and correct identification of pediatric NTCP deficiency can prevent excessive intervention and build a significant database for follow-up, as the long term prognosis of pediatric NTCP deficiency remains to be studied. As the incidence of NTCP deficiency is thought to be $0.64 \%$ in Han Chinese and 


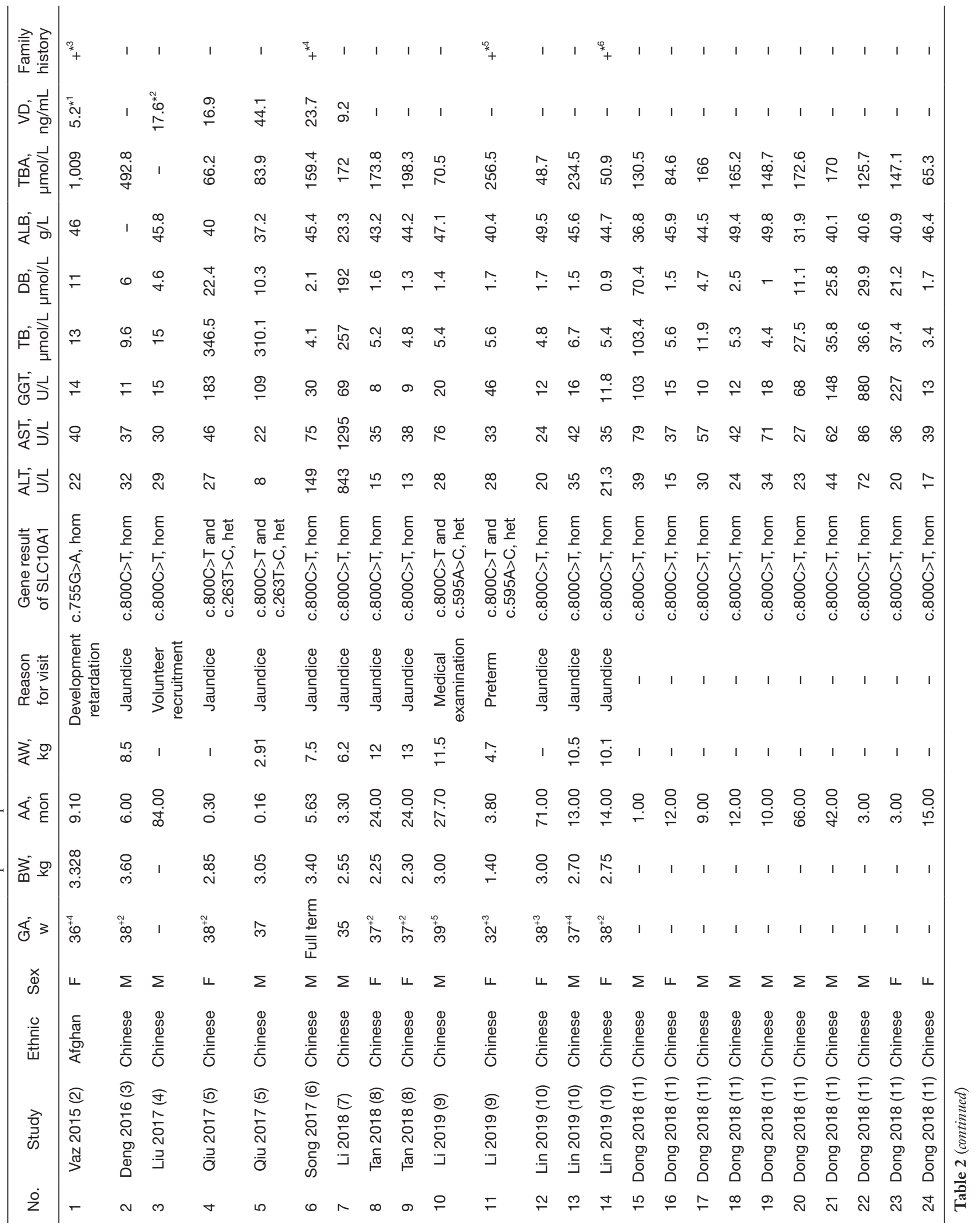




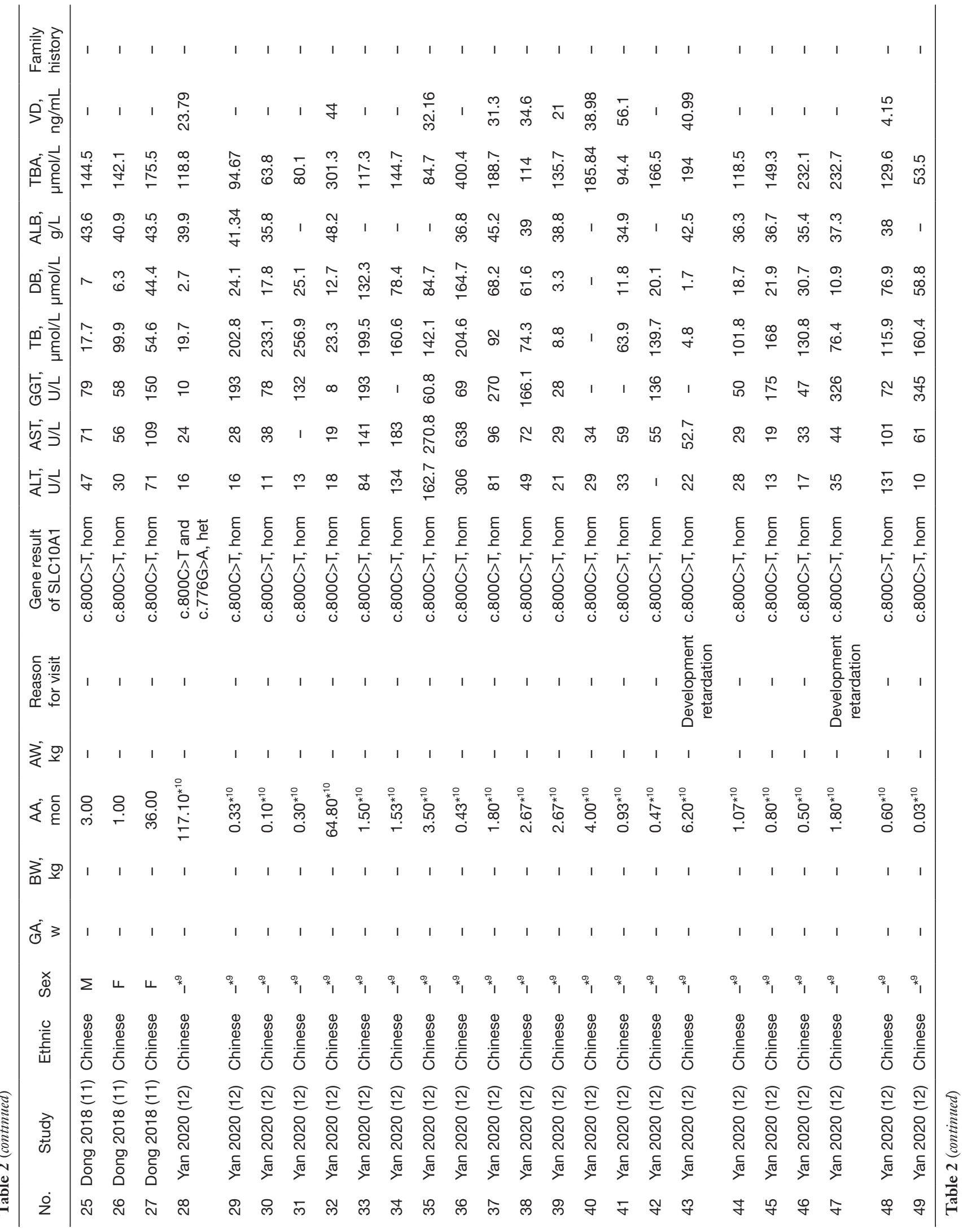




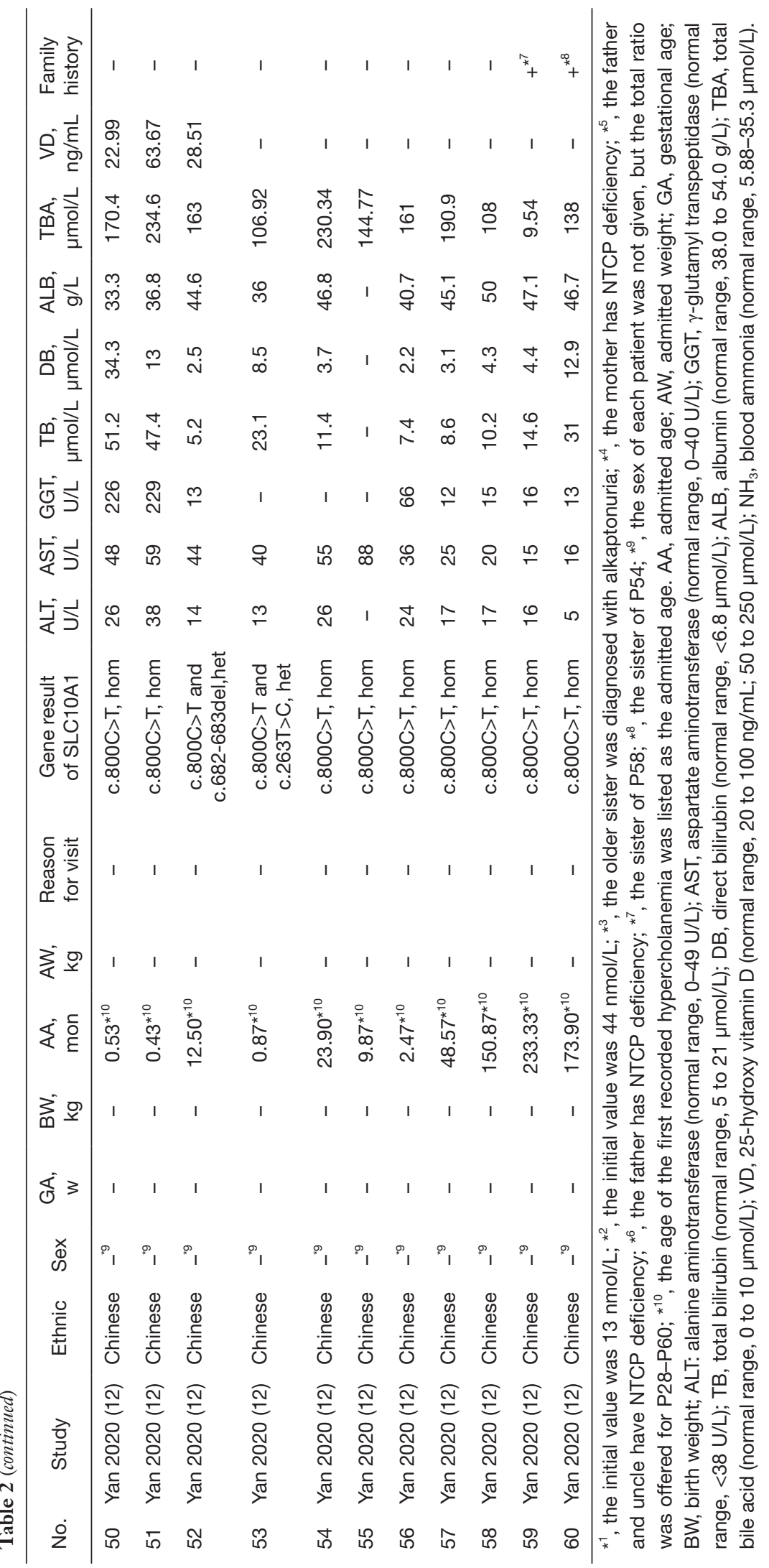


$1.44 \%$ in Dai Chinese (10), the majority of the cases in China have been missed. The jaundice is self-limiting and there are usually no other symptoms, and we inferred that another reason that it is not detected is that liver function tests at most hospitals do not routinely include TBA levels. Routine inclusion of TBA in the liver function test can detect hypercholanemia and identify patients for further screening and follow-up for NTCP deficiency.

To date, 71 cases of pediatric NTCP deficiency have been reported in Chinese children, and $64(90.1 \%)$ of them harbored homozygous mutations of c. $800 \mathrm{C}>\mathrm{T}$ in SLC10A1, which could represent the hot spot mutation of Chinese people. Screening for this mutation may therefore be useful as the primary genetic test for children with persistent hypercholanemia when infectious, structural, or immunological factors are excluded. Such screening could reduce the costs and be efficient.

In conclusion, the most common reason for visits of pediatric NTCP patients is jaundice, which is self-limiting. Pediatric NTCP deficiency might also be detected during routine check-ups or school physical examination if TBA is included with liver function tests. The hallmarks are hypercholanemia and mildly elevated AST, and screening for the c.800C $>$ T mutation in SLC1OA1 might be useful as the primary genetic test for Chinese children with persistent hypercholanemia after infectious, structural, immunological factors are excluded. A diagnosis of suspected pediatric NTCP deficiency can be made in children with self-limited hyperbilirubinemia and elevated AST in a setting of normal growth and development. Confirmation of the diagnosis requires genetic analysis for homozygous or compound heterozygous mutations of SLC10A1. Further study is needed to interpret the mechanisms of hyperbilirubinemia, elevated AST, and the absorption of fat soluble vitamins. We hope to have prolonged follow up with our patients, as the dynamic changes of childhood are important for identifying the pathogenesis and the prognosis of pediatric NTCP deficiency.

\section{Acknowledgments}

We are grateful to the patients and their parents for their cooperation and confidence during this study. We thank the BioMed Proofreading ${ }^{\circledR}$, LLC for language editing.

Funding: The present study was supported by a grant from the National Science and Technology Major Project of China (2018ZX10103001-003-006), Research Development Project of Sichuan Provincial Science and Technology
Department (No. 2020YFS0042), and Pediatric Clinical Research Center Foundation of Sichuan Province, China (No. 2017-46-4).

\section{Footnote}

Reporting Checklist: The authors have completed the CARE reporting checklist. Available at http://dx.doi.org/10.21037/ tp-20-360

Conflicts of Interest: All authors have completed the ICMJE uniform disclosure form (available at http://dx.doi. org/10.21037/tp-20-360). The authors have no conflicts of interest to declare.

Ethical Statement: The authors are accountable for all aspects of the work in ensuring that questions related to the accuracy or integrity of any part of the work are appropriately investigated and resolved. All procedures performed in studies involving human participants were in accordance with the ethical standards of the institutional and/or national research committee(s) and with the Helsinki Declaration (as revised in 2013). Written informed consent was obtained from the patient's legal guardian.

Open Access Statement: This is an Open Access article distributed in accordance with the Creative Commons Attribution-NonCommercial-NoDerivs 4.0 International License (CC BY-NC-ND 4.0), which permits the noncommercial replication and distribution of the article with the strict proviso that no changes or edits are made and the original work is properly cited (including links to both the formal publication through the relevant DOI and the license). See: https://creativecommons.org/licenses/by-nc-nd/4.0/.

\section{References}

1. Karpen SJ, Dawson PA. Not all (bile acids) who wander are lost: the first report of a patient with an isolated NTCP defect. Hepatology 2015;61:24-7.

2. Vaz FM, Paulusma CC, Huidekoper H, et al. Sodium taurocholate cotransporting polypeptide (SLC10A1) deficiency: conjugated hypercholanemia without a clear clinical phenotype. Hepatology 2015;61:260-7.

3. Deng $M$, Mao M, Guo L, et al. Clinical and molecular study of a pediatric patient with sodium taurocholate cotransporting polypeptide deficiency. Exp Ther Med 2016;12:3294-300. 
4. Liu R, Chen C, Xia X, et al. Homozygous p.Ser267Phe in SLC10A1 is associated with a new type of hypercholanemia and implications for personalized medicine. Sci Rep 2017;7:9214.

5. Qiu JW, Deng M, Cheng Y, et al. Sodium taurocholate cotransporting polypeptide (NTCP) deficiency: Identification of a novel SLC10A1 mutation in two unrelated infants presenting with neonatal indirect hyperbilirubinemia and remarkable hypercholanemia. Oncotarget 2017;8:106598-607.

6. Song YZ, Deng M. Sodium taurocholate cotransporting polypeptide deficiency manifesting as cholestatic jaundice in early infancy: a complicated case study. Zhongguo Dang Dai Er Ke Za Zhi 2017;19:350-4.

7. Li H, Qiu JW, Lin GZ, et al. Clinical and genetic analysis of a pediatric patient with sodium taurocholate cotransporting polypeptide deficiency. Zhongguo Dang Dai Er Ke Za Zhi 2018;20:279-84.

8. Tan HJ, Deng M, Qiu JW, et al. Monozygotic Twins Suffering From Sodium Taurocholate Cotransporting Polypeptide Deficiency: A Case Report. Front Pediatr 2018;6:354.

9. Li H, Deng M, Guo L, et al. Clinical and molecular characterization of four patients with NTCP deficiency from two unrelated families harboring the novel SLC10A1 variant c.595A>C (p.Ser199Arg). Mol Med Rep 2019;20:4915-24.

10. Lin H, Qiu JW, Rauf YM, et al. Sodium Taurocholate

Cite this article as: Zou TT, Zhu Y, Wan CM, Liao Q. Clinical features of sodium-taurocholate cotransporting polypeptide deficiency in pediatric patients: case series and literature review. Transl Pediatr 2021;10(4):1045-1054. doi: $10.21037 /$ tp-20-360
Cotransporting Polypeptide (NTCP) Deficiency Hidden Behind Citrin Deficiency in Early Infancy: A Report of Three Cases. Front Genet 2019;10:1108.

11. Dong C, Zhang BP, Wang H, et al. Clinical and histopathologic features of sodium taurocholate cotransporting polypeptide deficiency in pediatric patients. Medicine (Baltimore) 2019;98:e17305.

12. Yan YY, Wang MX, Gong JY, et al. Abnormal Bilirubin Metabolism in Patients With Sodium Taurocholate Cotransporting Polypeptide Deficiency. J Pediatr Gastroenterol Nutr 2020;71:e138-41.

13. Vaz FM, Huidekoper HH, Paulusma CC. Extended Abstract: Deficiency of Sodium Taurocholate Cotransporting Polypeptide (SLC10A1): A New Inborn Error of Metabolism with an Attenuated Phenotype. Dig Dis 2017;35:259-60.

14. Kadakol A, Ghosh SS, Sappal BS, et al. Genetic lesions of bilirubin uridine-diphosphoglucuronate glucuronosyltransferase (UGT1A1) causing CriglerNajjar and Gilbert syndromes: correlation of genotype to phenotype. Hum Mutat 2000;16:297-306.

15. Tazawa Y, Kobayashi K, Abukawa D, et al. Clinical heterogeneity of neonatal intrahepatic cholestasis caused by citrin deficiency: case reports from 16 patients. Mol Genet Metab 2004;83:213-9.

16. Van Herpe F, Waterham HR, Adams CJ, et al. NTCP deficiency and persistently raised bile salts: an adult case. J Inherit Metab Dis 2017;40:313-5. 
Table S1 The genes related to inherited metabolic liver disorders

\begin{tabular}{|c|c|c|c|c|c|c|c|}
\hline AARS2 & AASS & $A B C A 1$ & $A B C B 11$ & $A B C B 4$ & $A B C C 2$ & $A B C D 1$ & $A B C D 3$ \\
\hline ABCG5 & ABCG8 & ABHD5 & ACAD9 & $A C A D M$ & ACADS & $A C A D V L$ & ACAT1 \\
\hline ACOX 1 & ACOX2 & ACVRL1 & $A D A$ & $A D A 2$ & ADAMTSL2 & $A D K$ & $A G A$ \\
\hline$A G L$ & AGPAT2 & AGPS & $A G X T$ & $\mathrm{AHCY}$ & AIFM1 & AIRE & AKR1D1 \\
\hline AKT2 & $A L A D$ & ALAS2 & ALDH2 & $A L D O A$ & $A L D O B$ & ALG1 & ALG11 \\
\hline ALG12 & ALG13 & ALG2 & ALG3 & ALG6 & ALG8 & ALG9 & ALMS1 \\
\hline$A L P L$ & $A M A C R$ & AMT & ANGPTL3 & ANK1 & ANKS6 & AP1S1 & APCS \\
\hline$A P O A 1$ & APOA5 & $A P O E$ & APOPT1 & ARG1 & ARSA & $A R S B$ & $A S A H 1$ \\
\hline$A S L$ & ASS1 & ATP13A2 & ATP5A1 & ATP6AP1 & ATP7B & ATP8B1 & ATPAF2 \\
\hline$B 2 M$ & B4GALT1 & BAAT & $B C A P 31$ & $B C K D H A$ & $B C K D H B$ & $B C S 1 L$ & BLVRA \\
\hline BMP2 & BMPER & BOLA3 & BRAF & BSCL2 & BTD & C12orf65 & C15ORF41 \\
\hline$C 1 Q B P$ & CALM1 & CALM2 & CALM3 & CARS2 & CASP10 & CAT & CAVIN1 \\
\hline CBS & $C C 2 D 2 A$ & CCDC115 & CCND1 & CD4OLG & CDAN1 & CDKN1C & CEP164 \\
\hline CEP19 & CEP41 & CEP83 & CFAP53 & CFC1 & CFTR & CIITA & CLDN1 \\
\hline CLN3 & CLN5 & CLN6 & CLN8 & COA7 & COG1 & COG2 & COG4 \\
\hline COG5 & COG6 & COG7 & COG8 & COQ2 & $\operatorname{cox} 10$ & $\operatorname{cox} 14$ & $\operatorname{cox} 15$ \\
\hline COX20 & COX6B1 & COX8A & $C P$ & CPOX & CPS1 & CPT1A & CPT2 \\
\hline CSPP1 & CTC1 & CTLA4 & CTNS & CTSC & CTSD & CTSF & CYBA \\
\hline CYC1 & CYP27A1 & CYP7B1 & $D B T$ & $D C D C 2$ & DCLRE1C & $D D C$ & DDOST \\
\hline DGUOK & DHCR7 & DHDDS & DHFR & $D K C 1$ & DLD & DNAJC5 & DOLK \\
\hline DPAGT1 & DPM1 & DPM2 & DPM3 & DPYS & DYNC2H1 & DYNC2LI1 & EARS2 \\
\hline EIF2AK3 & ELAC2 & $E N G$ & ENO3 & $E P H X 1$ & EPM $2 A$ & ETFA & ETFB \\
\hline ETFDH & EXTL3 & F8 & F9 & $F A D D$ & $F A H$ & FAM111A & FAN1 \\
\hline FARS2 & FAS & FASLG & FASTKD2 & $F B P 1$ & FBXL44 & FCGR2A & $F E C H$ \\
\hline FERMT3 & $F G A$ & $F G B$ & $F G G$ & $F H$ & FIG4 & FOXRED1 & FTH1 \\
\hline FUCA1 & FXN & G6PC & G6PC3 & $G 6 P D$ & GAA & GALC & GALE \\
\hline GALK1 & GALNS & GALT & GANAB & GATA6 & GBA & GBE1 & $\mathrm{GCH} 1$ \\
\hline GCSH & GDF1 & GDF2 & GFM1 & GLA & GLB1 & $G L D C$ & GLIS2 \\
\hline GLIS3 & GLUD1 & GM2A & GNE & GNMT & GNPAT & GNPTAB & GNPTG \\
\hline GPD1 & GRN & GSN & GSTZ1 & GUCY2D & GUSB & GYG1 & GYS1 \\
\hline GYS2 & $H 19$ & $H A D H$ & $H A D H A$ & $H A D H B$ & HAMP & $H B B$ & HEXA \\
\hline HEXB & HFE & HFE2 & $H G D$ & HGSNAT & HLA-DRB1 & HMBS & HMGCL \\
\hline HNF1B & HNF4A & $H P D$ & HSD17B4 & HSD3B7 & HYAL1 & IARS & IDS \\
\hline IDUA & IER3IP1 & IFT122 & IFT140 & IFT172 & IFT43 & IFT80 & IFT81 \\
\hline IL21R & IL2RA & IL31RA & IL7R & INVS & $I T C H$ & ITK & JAG1 \\
\hline KCNH1 & KCNQ1OT1 & KCTD7 & KHK & KIAA0586 & KRAS & KRT18 & KRT8 \\
\hline KYNU & LAMP2 & LARS & LARS2 & $\angle B R$ & LDHA & $\angle D H B$ & LIPA \\
\hline LIPF & LIPI & LIPT1 & LMNA & $L P L$ & $\angle R P P R C$ & LYRM4 & LYRM7 \\
\hline$L Y Z$ & LZTR1 & MAN2B1 & MANBA & MARS & MAT1A & MCEE & MCOLN1 \\
\hline MDH2 & MEGF8 & MFSD 8 & MGAT2 & MGME1 & MKS1 & MMAA & $M M A B$ \\
\hline MMACHC & $M M A D H C$ & MMP21 & MOGS & MPDU1 & $M P I$ & MPV17 & MRPL3 \\
\hline MRPL44 & MRPS16 & MRPS22 & MRPS7 & MT1B & MTFMT & MTHFD1 & MTO1 \\
\hline MTTP & MUC5B & MUT & $M V K$ & MYO5B & NAGA & NAGLU & NAGS \\
\hline NARS2 & NAT2 & NBAS & NCF1 & NCF2 & NDUFA10 & NDUFA12 & NDUFA4 \\
\hline NDUFA9 & NDUFAF2 & NDUFAFG & NDUFS3 & NDUFS7 & NDUFS8 & NEK1 & NEK8 \\
\hline NEU1 & NFE2L2 & $N G L Y 1$ & NHLRC1 & NHP2 & NOP10 & NOTCH2 & NPC1 \\
\hline NPC2 & NPHP1 & NPHP3 & NPHP4 & NR1H4 & NSMCE2 & OCLN & OFD1 \\
\hline OSMR & OTC & $P A H$ & $P C$ & $P C C A$ & $P C C B$ & PCK1 & PDHA1 \\
\hline PET100 & PEX1 & PEX10 & PEX11B & PEX12 & PEX13 & PEX14 & PEX16 \\
\hline PEX19 & PEX2 & PEX26 & PEX3 & PEX5 & PEX6 & PEX7 & PFKM \\
\hline PGAM2 & PGK1 & PGM1 & PHKA1 & PHKA2 & PHKB & PHKG1 & PHKG2 \\
\hline PHYH & PIBF1 & PIEZO1 & $P I G A$ & PKD1 & PKD2 & PKHD1 & $P K L R$ \\
\hline PMM2 & PNPLA2 & PNPT1 & POLD1 & $P O L G$ & POLG2 & $P O M C$ & PPARG \\
\hline PPT1 & PRDX1 & PRF1 & PRKAG2 & PRKCSH & $P R O D H$ & $P S A P$ & PSMB8 \\
\hline PTF1A & PTPN11 & PTPRC & PTS & PYGL & PYGM & RAF1 & RAG1 \\
\hline RAG2 & RBCK1 & RFT1 & $R F X 5$ & $R F X 6$ & RFXANK & $R F X A P$ & RHAG \\
\hline RIT1 & $R M N D 1$ & RNASEH2A & RPGRIP1L & $R R M 2 B$ & SAMHD1 & SAR1B & $S C 5 D$ \\
\hline SCN9A & SCO1 & SCP2 & $S C Y L 1$ & $S D H A$ & SDHAF1 & $S D H D$ & SEC63 \\
\hline SERAC1 & SERPINA1 & SFTPA1 & SFTPA2 & SFXN4 & SGSH & $S H 2 D 1 A$ & SHANK3 \\
\hline SKIV2L & SLC10A1 & SLC10A2 & SLC11A2 & SLC17A5 & $S L C 22 A 5$ & SLC25A13 & SLC25A15 \\
\hline SLC25A20 & SLC25A4 & SLC27A5 & SLC29A3 & $S L C 2 A 2$ & SLC30A10 & SLC35A1 & SLC35A2 \\
\hline SLC35C1 & SLC37A4 & $S L C 39 A 8$ & SLC40A1 & SLC52A3 & SLC6A19 & SLC7A7 & SLCO1B1 \\
\hline SLCO1B3 & SMPD1 & sos1 & sos2 & SP110 & SPRTN & SRD5A3 & ST3GAL5 \\
\hline STN1 & STTЗA & STT3B & STX11 & STXBP2 & SUCLA2 & SUCLG1 & SUMF1 \\
\hline SURF1 & SYNJ1 & TACO1 & TALDO1 & TARS2 & TAT & $T A Z$ & TCIRG1 \\
\hline TCTEX1D2 & TERC & TERT & $T F$ & TFAM & TFR2 & TGFB1 & TJP2 \\
\hline TK2 & TMEM107 & TMEM165 & TMEM199 & TMEM216 & TMEM67 & TPP1 & TRAF3IP1 \\
\hline TRAPPC11 & TREX1 & TRIM37 & TRMT10C & TRMU & TSFM & TTC21B & ТТС37 \\
\hline TTPA & $T T R$ & TUFM & $T W N K$ & UBR1 & UGT1A1 & UNC13D & UPB 1 \\
\hline UQCC2 & UQCRB & UQCRC2 & UQCRQ & UROC1 & UROD & UROS & USP18 \\
\hline UTP4 & VARS2 & $V H L$ & VIL1 & VIPAS39 & VPS33B & WDR19 & WDR34 \\
\hline WDR35 & WDR60 & XPNPEP3 & YARS2 & ZNF423 & & & \\
\hline
\end{tabular}




\begin{tabular}{|c|c|c|c|c|c|c|c|c|c|c|c|c|c|}
\hline No. & $\begin{array}{l}\text { Time, yyyy. } \\
\text { mm.dd }\end{array}$ & $\begin{array}{c}\text { TBA, } \\
\mu \mathrm{mol} / \mathrm{L}\end{array}$ & $\begin{array}{l}\text { ALT, } \\
\text { U/L }\end{array}$ & $\begin{array}{l}\text { AST, } \\
\text { U/L }\end{array}$ & $\begin{array}{l}\text { GGT, } \\
\text { U/L }\end{array}$ & $\begin{array}{c}\mathrm{TB}, \\
\mu \mathrm{mol} / \mathrm{L}\end{array}$ & $\begin{array}{c}\mathrm{DB}, \\
\mu \mathrm{mo} / \mathrm{L}\end{array}$ & $\begin{array}{c}\mathrm{BU}, \\
\mu \mathrm{mol} / \mathrm{L}\end{array}$ & $\begin{array}{l}\text { ALB, } \\
g / L\end{array}$ & $\begin{array}{c}\mathrm{TG}, \\
\mu \mathrm{mol} / \mathrm{L}\end{array}$ & $\begin{array}{c}\mathrm{TC}, \\
\mu \mathrm{mol} / \mathrm{L}\end{array}$ & $\begin{array}{c}\mathrm{VD}, \\
\mathrm{ng} / \mathrm{mL}\end{array}$ & $\begin{array}{c}\mathrm{NH} 3, \\
\mu \mathrm{mol} / \mathrm{L}\end{array}$ \\
\hline \multirow[t]{8}{*}{$\mathrm{P} 1$} & 2018.08.25 & 170.0 & 34 & 47 & 37 & 38.5 & 12.8 & 25.7 & 44.9 & & & & \\
\hline & 2018.10.09 & 90.3 & 43 & 54 & 23 & 22.5 & 7.2 & 15.3 & 46.6 & & & & \\
\hline & 2018.11.19 & 108.0 & 43 & 62 & 18 & 13.4 & 4.6 & 8.8 & 51.8 & & & & \\
\hline & 2018.12.20 & 105.9 & 37 & 64 & 14 & 17.1 & 5.0 & 12.1 & 54.8 & & & & \\
\hline & 2018.12.26 & 104.2 & 30 & 43 & 12 & 11.9 & 4.4 & 7.5 & 45.3 & 0.79 & 4.15 & 36.8 & 13.0 \\
\hline & 2019.01.20 & 103.7 & 32 & 59 & 13 & 9.4 & 3.8 & 5.6 & 50.8 & & & & \\
\hline & 2019.03.26 & 96.7 & 32 & 52 & 15 & 13.1 & 4.2 & 8.9 & 52.1 & & & & \\
\hline & 2020.09.09 & 120.5 & 25 & 39 & 9 & 8.8 & 3.8 & 5.0 & 44.6 & & & & \\
\hline \multirow[t]{9}{*}{ P2 } & 2016.07.12 & 107.6 & 13 & 35 & 9 & 8.9 & 4.3 & 4.6 & 45.2 & & & & \\
\hline & 2016.08.29 & 128.7 & 9 & 29 & 9 & 16.5 & 7.9 & 8.6 & 45.4 & & & & \\
\hline & 2019.02.25 & 151.6 & 16 & 33 & 8 & 19.0 & 7.0 & 12.0 & 43.5 & & & & \\
\hline & 2019.03.07 & - & 10 & 24 & 9 & 9.3 & 3.8 & 5.5 & 41.8 & 0.7 & 2.7 & & 24 \\
\hline & 2019.03 .08 & 148.1 & & & & & & & & & & & \\
\hline & 2019.05 .23 & 256.4 & 8 & 26 & 9 & 11.8 & 4.3 & 7.5 & 42.9 & & & & \\
\hline & 2019.06.27 & 71.8 & 9 & 26 & 7 & 26.1 & 9.0 & 17.1 & 43.1 & & & & \\
\hline & 2019.08.02 & 159.3 & 6 & 23 & 11 & 17.1 & 6.8 & 10.3 & 44.2 & & & & \\
\hline & 2020.04 .19 & 90.4 & 8 & 29 & 8 & 27.1 & 8.4 & 18.7 & 47.1 & & & & \\
\hline \multirow[t]{9}{*}{ P3 } & 2018.08.12 & 69.4 & 30 & 42 & 23 & 29.5 & 9.3 & 20.2 & 41.7 & & & & \\
\hline & 2018.12.12 & 158.7 & 29 & 51 & 11 & 10.5 & 4.5 & 6.0 & 48.8 & & & & \\
\hline & 2019.01.16 & 104.8 & 13 & 39 & 11 & 10.8 & 3.4 & 7.4 & 47.3 & & & & \\
\hline & 2019.02.22 & 21.2 & 19 & 45 & 8 & 12.1 & 4.4 & 7.7 & 47.8 & & & & \\
\hline & 2019.03.20 & 24.2 & 17 & 39 & 12 & 7.0 & 1.4 & 5.6 & 45.2 & & & & \\
\hline & 2019.04.19 & 49.4 & 16 & 43 & 10 & 8.3 & 2.6 & 5.7 & 48.8 & & & & \\
\hline & 2019.04 .23 & 41.7 & 13 & 41 & 9 & 5.5 & 2.3 & 3.2 & 47.6 & & & & 48 \\
\hline & 2019.08.22 & 79.2 & 26 & 44 & 10 & 8.7 & 3.3 & 5.4 & 47.0 & & & & \\
\hline & 2021.01.09 & 29.7 & 11 & 37 & 12 & 10.1 & 2.5 & 7.6 & 54 & & & & \\
\hline \multirow[t]{3}{*}{ P4 } & 2019.04.25 & 180 & 75 & 112 & 249 & 79.6 & & 21.6 & 33.6 & & & $<8.0$ & 49 \\
\hline & 2019.05.03 & 212.9 & 37 & 68 & 316 & 39.1 & 31.0 & 8.1 & 34.4 & & & & \\
\hline & 2019.05 .25 & - & 15 & 32 & 95 & 11.9 & & 4.9 & 40.3 & & & 22.5 & 47 \\
\hline \multirow[t]{3}{*}{ P5 } & 2020.02.26 & 297.6 & 36 & 65 & - & 81.2 & 5.4 & 75.8 & - & & & & \\
\hline & 2020.03.04 & 162.4 & 29 & 36 & - & 41.4 & 2.4 & 39.0 & 42.2 & & & & \\
\hline & 2020.04 .03 & 228.8 & 17 & 37 & - & 28.6 & 7.7 & 20.9 & 42.2 & & & & \\
\hline \multirow[t]{8}{*}{ P6 } & 2019.01.07 & 57.5 & 64 & 82 & 25 & 12.9 & 5.2 & 7.7 & 54.0 & & & & \\
\hline & 2019.02.27 & 42.2 & 28 & 41 & 18 & 8.3 & 3.1 & 5.2 & 47.3 & & & & \\
\hline & 2019.03.26 & 66.9 & 28 & 47 & 13 & 5.2 & 2.1 & 3.1 & 46.4 & & & & \\
\hline & 2019.04.28 & 35.3 & 15 & 39 & 16 & 3.5 & 1.6 & 1.9 & 42.5 & & & & \\
\hline & 2019.05 .28 & 24.5 & 38 & 66 & 16 & 9.3 & 2.9 & 6.4 & 46.8 & & & & \\
\hline & 2019.06.27 & 29.7 & 23 & 39 & 12 & 10.7 & 3.4 & 7.3 & 43.3 & & & & \\
\hline & 2019.07.25 & 32.5 & 19 & 47 & 10 & 7.3 & 2.1 & 5.2 & 44.4 & & & & \\
\hline & 2021.01.09 & 6.1 & 20 & 46 & 13 & 7.1 & 0.01 & 7.09 & 45.5 & & & 27.26 & \\
\hline \multirow[t]{5}{*}{ P7 } & 2018.12.29 & 74.1 & 26 & 43 & 90 & 30.8 & 13.1 & 17.7 & 35.4 & & & & \\
\hline & 2019.03.01 & 123.1 & 35 & 49 & 24 & 12.7 & 4.8 & 7.9 & 49.1 & & & & \\
\hline & 2019.04.14 & 76.0 & 33 & 45 & 15 & 6.6 & 2.9 & 3.7 & 42.9 & & & & \\
\hline & 2019.06.04 & 31.2 & 64 & 65 & 17 & 7.9 & 2.6 & 5.3 & 47.3 & & & & \\
\hline & 2019.08 .12 & 49.6 & 19 & 40 & $<6$ & 7.6 & 2.8 & 4.8 & 41.9 & & & & \\
\hline \multirow[t]{2}{*}{ P8 } & 2019.09.24 & 93.3 & 10 & 26 & $<6$ & 8.0 & 2.4 & 5.6 & 43.0 & & & & \\
\hline & 2020.09.29 & 47.9 & 12 & 31 & 9 & 13.6 & 2.5 & 11.1 & 43.9 & & & & \\
\hline \multirow[t]{5}{*}{ P9 } & 2019.07.07 & 82.6 & 39 & 53 & 26 & 11.1 & 3.3 & 7.8 & 40.1 & & & & \\
\hline & 2019.08.15 & 60.4 & 37 & 53 & 13 & 9.2 & 2.8 & 6.4 & 43.8 & & & & \\
\hline & 2019.09.19 & 85.8 & 42 & 60 & 11 & 7.1 & 2.6 & 4.5 & 46.5 & & & & \\
\hline & 2019.10.20 & 117.3 & 34 & 57 & 13 & 8.7 & 3.1 & 5.6 & 51.7 & & & & \\
\hline & 2020.08 .28 & - & 19 & 45 & 7 & 9.5 & 2.7 & 6.8 & 49.0 & & & & \\
\hline \multirow[t]{2}{*}{ P10 } & 2019.12.27 & 43.1 & 21 & 37 & 16 & 11.5 & 5.3 & 6.2 & 44.5 & 0.77 & 2.73 & & 36.5 \\
\hline & 2021.01.05 & 97.99 & 19.7 & 41.5 & 11.6 & 12.1 & 4.9 & 7.2 & 42.3 & & & 28.86 & \\
\hline \multirow[t]{2}{*}{ P11 } & 2018.09.19 & 107.6 & 18 & 44 & 15 & 7.3 & 2.0 & 5.3 & 46.6 & & & & \\
\hline & 2020.03 .18 & 86.3 & 10 & 29 & 8 & 4.4 & 1.3 & 3.1 & 35.4 & 1.16 & 3.66 & 27.3 & \\
\hline P12 & 2020.04 .10 & 26.5 & 31 & 45 & 9 & 11.2 & 3.0 & 8.2 & 43.8 & & & & \\
\hline & 2020.04.20 & 75.1 & 25 & 43 & 7 & 7.3 & 2.3 & 5.0 & 45.4 & & & & \\
\hline & 2020.05.22 & 51.2 & 22 & 44 & 10 & 8.1 & 2.8 & 5.3 & 45.8 & & & & \\
\hline & 2020.07.17 & 80.3 & 44 & 54 & 17 & 10.6 & 3.3 & 7.3 & 47.6 & & & & \\
\hline & 2020.07.21 & 71.9 & 23 & 42 & 12 & 6.0 & 1.7 & 4.3 & 46.7 & & & 33.7 & \\
\hline
\end{tabular}

